\title{
Características de Crianças com Altas Habilidades/ Superdotação: UMA REVISÃo SisTEMÁTICA ${ }^{1}$
}

\author{
CharaCteristics of GIFTED CHILDREN: A SySTEMATIC REVIEW
}

\author{
Maira Maria da COSTA ${ }^{2}$ \\ Alessandra Sant'Anna BIANCHI ${ }^{3}$ \\ Márcia Melo de Oliveira SANTOS ${ }^{3}$
}

\begin{abstract}
RESUMO: Este estudo de revisão sistemática teve como objetivo identificar as características de crianças com altas habilidades/ superdotação. Os critérios de inclusão foram: artigos científicos sobre pesquisas empíricas, publicados no período de 2010 a 2019 , com participantes com altas habilidades/superdotação, menores de 12 anos, e a avaliação de altas habilidades/superdotação deveria ter, pelo menos, um teste de inteligência associado a outros instrumentos. Os critérios de exclusão foram: nenhum grupo composto apenas por crianças com altas habilidades/superdotação, não apresentar resultados exclusivos às crianças com altas habilidades/ superdotação ou o grupo de crianças com altas habilidades/superdotação ter participantes com dupla excepcionalidade, deficiência física ou sensorial. A busca dos artigos foi feita nas bases Scopus e Web of Science em janeiro de 2020 e SciELO.org em abril de 2020. Foram analisados 29 artigos, agrupados em cinco categorias. Os resultados são apresentados em síntese narrativa e confirmam o caráter heterogêneo das altas habilidades/superdotaçáo. A maioria dos artigos explorou características da cognição e dos processos de identificação e avaliação das crianças. Dentre as principais limitações, estâo a obtençâo de artigos com autores repetidos e a obtençáo incompleta dos artigos potencialmente relevantes. Esta pesquisa contribui para a visibilidade sobre as características de crianças com superdotação, com um enfoque geral e amplo.
\end{abstract}

PALAVRAS-CHAVE: Pessoa com necessidades especiais. Educação especial. Inclusão. Aptidôes. Inteligência.

\begin{abstract}
This systematic review study aimed to identify the characteristics of gifted children. Inclusion criteria were: scientific articles on empirical research published in the period from 2010 to 2019, whose participants were gifted children under 12 years old, and the evaluation of giftedness should have, at least, one intelligence test associated with other instruments. The exclusion criteria were: no group consisting of only gifted children, no exclusive results presented to gifted children, or the group of gifted children have participants with double exceptionality, physical or sensory disabilities. The search for articles was made in Scopus and Web of Science databases in January 2020 and SciELO.org in April 2020. Twenty-nine articles were analyzed, grouped into five categories. The results are presented in narrative synthesis, and confirm the heterogeneous feature of giftedness. Most articles explored characteristics of children's cognition and identification and assessment processes. Among the main limitations are the obtaining of articles with repeated authors and incomplete obtaining of potentially relevant articles. This research contributes to the visibility of the characteristics of gifted children, with a general and broad focus.
\end{abstract}

KEYWORDS: Person with special needs. Special education. Inclusion. Aptitudes. Intelligence.

\section{INTRODUÇÁo}

A pessoa que apresenta altas habilidades/superdotação (AH/SD) tem desempenho superior, em comparaçáo aos seus pares, em uma ou mais áreas do conhecimento (Cupertino, 2008). As habilidades podem ser expressas na capacidade intelectual geral, em aptidáo acadêmica, na criatividade, em capacidade de liderança, talento para as artes e na capacidade

\footnotetext{
1 https://doi.org/10.1590/1980-54702022v28e0121
}

2 Psicóloga da Prefeitura Municipal de Jaraguá do Sul. Mestre em Psicologia - Universidade Federal do Paraná - UFPR. Especialização em Neuropsicologia Clínica e em Saúde da Família. Jaraguá do Sul/Santa Catarina/Brasil. E-mail: mairadacostapsico@gmail. com. ORCID: https://orcid.org/0000-0001-8696-190X

3 Professora na Universidade Federal do Paraná - UFPR. Curitiba/Paraná/Brasil. E-mail: bianchi@ufpr.br. ORCID: https://orcid. org/0000-0003-3937-4520. Mestranda. Pós-Graduação em Psicologia. Universidade Federal do Paraná - UFPR. Curitiba/Paraná/Brasil. E-mail: psi_mmarcia@hotmail.com. ORCID: https://orcid.org/0000-0002-9105-8325 
psicomotora (Virgolim, 2007). Identificar sujeitos com AH/SD tem sua devida importância a partir do momento em que se compreende que essas pessoas são diferentes em diversos aspectos e têm necessidades educacionais diferentes, que exigem estratégias de ensino diferenciadas (Cupertino, 2008; Virgolim, 2007). Nas crianças com AH/SD, o desenvolvimento é atípico, elas apresentam domínios cognitivos em idade anterior à média e progridem rapidamente na aprendizagem (Winner, 1998). No entanto, a maturidade emocional pode náo acompanhar o rápido progresso cognitivo, causando uma dessincronia no desenvolvimento (Terrassier, 2009).

No ano de 2020, na Educação Básica brasileira, havia 24.132 estudantes com altas habilidades/superdotação, de acordo com o Censo Escolar (Instituto Nacional de Estudos e Pesquisas Educacionais Anísio Teixeira [INEP], 2020), o que representa apenas 0,05\% do total de alunos matriculados. Nos Estados Unidos da América, no ano de 2016, a incidência de alunos com AH/SD era de 6,45\% do total de alunos matriculados nas escolas públicas (Civil rights data collection, 2016). As taxas brasileiras sugerem que, no país, há dificuldades na identificação das crianças e adolescentes com AH/SD. Para Barreto e Mettrau (2011), é fundamental a difusão do conhecimento acerca das AH/SD no campo educacional, abordando não somente características desses alunos ou conceitos sobre o tema, mas também os professores devem ter acesso a informaçóes sobre os alunos, direcionadas ao processo de ensino-aprendizagem que promovam práticas educacionais que contribuam para o crescimento acadêmico, motivacional e criativo.

Diversos autores (por exemplo, Cupertino, 2008; Renzulli, 2005; Winner, 1998) destacam a importância do ambiente educativo diferenciado para os alunos com AH/SD. A identificação das crianças com AH/SD possibilita que tenham a oportunidade de receber o atendimento especializado de que precisam, que impulsiona o desenvolvimento da criatividade e do interesse em aprender (Piske et al., 2016). O conhecimento das características dessas crianças contribui para a compreensão do fenômeno da superdotação e para a implementação de açóes voltadas às necessidades específicas delas. De acordo com Cupertino (2008), o conhecimento das características da superdotação contribui para a identificação, a avaliação diagnóstica e para a prática educativa. Evidencia-se, ainda, uma lacuna na formação dos professores para o trabalho com esses alunos (Barreto \& Mettrau, 2011), conforme discutido por Bahiense e Rossetti (2014), além da formação deficitária, professores também apresentam representações das AH/SD que dizem respeito aos mitos da superdotação. É essencial, portanto, a difusão do conhecimento sobre aspectos reais desses sujeitos, para que os professores possam planejar a prática pedagógica em consonância com as verdadeiras necessidades dos alunos. Assim, este estudo teve o objetivo geral de identificar as características de crianças com altas habilidades/superdotação no intuito de responder à pergunta: Quais são as características de crianças com altas habilidades/superdotação conforme as pesquisas empíricas publicadas no período de 2010 a 2019?

\section{Método}

Esta revisão sistemática é relatada conforme a recomendação PRISMA (Page et al., 2021). Foi delineada seguindo as etapas de delimitação da questão pesquisada, escolha das fontes de dados, definição das palavras-chave para a busca, busca e armazenamento dos resultados, seleção dos artigos conforme os critérios de elegibilidade, extração dos dados dos artigos selecionados e síntese e interpretação dos dados (Costa \& Zoltowski, 2014). 


\subsection{Critérios de Elegibilidade}

São critérios de inclusão: os participantes serem crianças com AH/SD; os participantes devem ser menores de 12 anos; os artigos devem ter sido publicados no período de 2010 a 2019; serem artigos científicos relatando pesquisas empíricas; a avaliação de AH/SD das crianças que compóem a amostra deve ter incluído pelo menos um teste de inteligência associado a outros instrumentos de investigação utilizados na avaliação de AH/SD, tais como testes de criatividade, escalas de comportamento de superdotação, de habilidades, talentos e alto desempenho, de desempenho escolar, nomeação por pais, professores e alunos, inventários, escalas e/ou entrevistas respondidas por pais, professores e/ou a própria criança que investigam indicadores de AH/SD. Ressalta-se que esse outro instrumento de investigação não pode ser, também, um teste de inteligência. $\mathrm{O}$ critério de idade foi definido considerando o limite de idade entre infância e adolescência definido no Brasil pelo Estatuto da Criança e do Adolescente (Lei no 8.069, de 13 de julho de 1990). Foram incluídos apenas os artigos que apresentavam a idade dos participantes. O critério adotado sobre a avaliação de AH/SD requer que as crianças tenham sido identificadas em um processo de avaliação que não se limita ao resultado do teste de inteligência. São critérios de exclusão: nenhum grupo da amostra ser composto apenas por crianças com AH/SD; não apresentar resultados que sejam exclusivos ao grupo de crianças com $\mathrm{AH} / \mathrm{SD}$; o grupo de crianças com $\mathrm{AH} /$ SD ser composto por crianças com dupla excepcionalidade (dificuldade de aprendizagem, atraso global do desenvolvimento, deficiência intelectual, transtorno do espectro autista, transtornos motores, transtornos da comunicação, transtornos da aprendizagem, transtorno do déficit de atenção e hiperatividade, transtornos psiquiátricos); o grupo de crianças com AH/SD ser constituído por crianças com deficiência física ou sensorial. Os artigos que não apresentaram resultados exclusivos ao grupo de crianças com $\mathrm{AH} / \mathrm{SD}$, ou nos quais não havia pelo menos um grupo de amostra formado por crianças com AH/SD, foram excluídos, assim como os artigos nos quais as amostras eram compostas por crianças com dupla excepcionalidade, considerando que há especificidades que não devem ser estendidas às crianças com AH/SD sem dupla excepcionalidade. Esse critério contribui para garantir a seleção de pesquisas com amostras mais parecidas. Conforme definição de Pfeiffer (2015), pessoas com dupla excepcionalidade são aquelas que apresentam AH/ SD juntamente a algum distúrbio psiquiátrico, deficiências ou dificuldades de aprendizagem.

\subsection{EsTRATÉGIA DE BUSCA E SELEÇÃo DOS ESTUdOS}

As palavras-chave utilizadas na busca foram "gifted" e "child”. A pesquisa nas bases de dados foi feita utilizando as palavras-chave e o operador booleano "AND”, a string de pesquisa utilizada foi gifted ${ }^{*} A N D$ child $^{*}$. Foi aplicada delimitação temporal, restringindo as publicações do período entre 2010 e 2019 e não foi utilizado critério de delimitação do idioma. A busca foi realizada nas bases de dados eletrônicas Scopus e Web of Science, em 13 de janeiro de 2020 e SciELO.org em 29 de abril de 2020. Todo o processo de busca das publicaçóes foi realizado por dois juízes, de forma independente, obtendo-se os mesmos resultados.

$\mathrm{Na}$ etapa de seleção, todos os títulos, resumos, palavras-chave, autores e periódicos das referências encontradas foram salvos, totalizando 2.211 referências potencialmente relevantes. A seguir foi realizada a exclusão das referências duplicadas. A seleção inicial, feita com base na leitura dos resumos, de acordo com os critérios de elegibilidade, foi realizada por dois juízes, de forma independente. A concordância entre os juízes foi superior a $90 \%$, nos casos em 
que houve divergência, os artigos foram incluídos na etapa seguinte, para leitura na íntegra. Ao final dessa etapa inicial, foram selecionados 303 artigos. Dentre os artigos selecionados, a oito deles não foi possível o acesso, pois, mesmo presentes nas bases de dados, não estavam disponíveis no portal de Periódicos da Coordenação de Aperfeiçoamento de Pessoal de Nível Superior (CAPES). Devido à pandemia de Covid-19, o serviço de comutação bibliográfica estava indisponível. Foi, portanto, enviado e-mail aos autores, mas não se obteve retorno. Considerando que não se obteve acesso ao texto integral dos artigos, eles foram eliminados nessa etapa, restando 295 artigos para leitura na íntegra. Feita a nova verificação da elegibilidade, a partir da leitura do artigo completo, restaram 29 artigos elegíveis. Esse processo de seleção foi realizado, de forma independente, por dois juízes. Nos casos em que houve divergência, foi realizada reunião de consenso, para discussão e verificação dos critérios de elegibilidade, se eram atendidos ou não. A Figura 1 apresenta o fluxo de seleção dos artigos.

\section{Figura 1}

Fluxo de seleção dos artigos relevantes

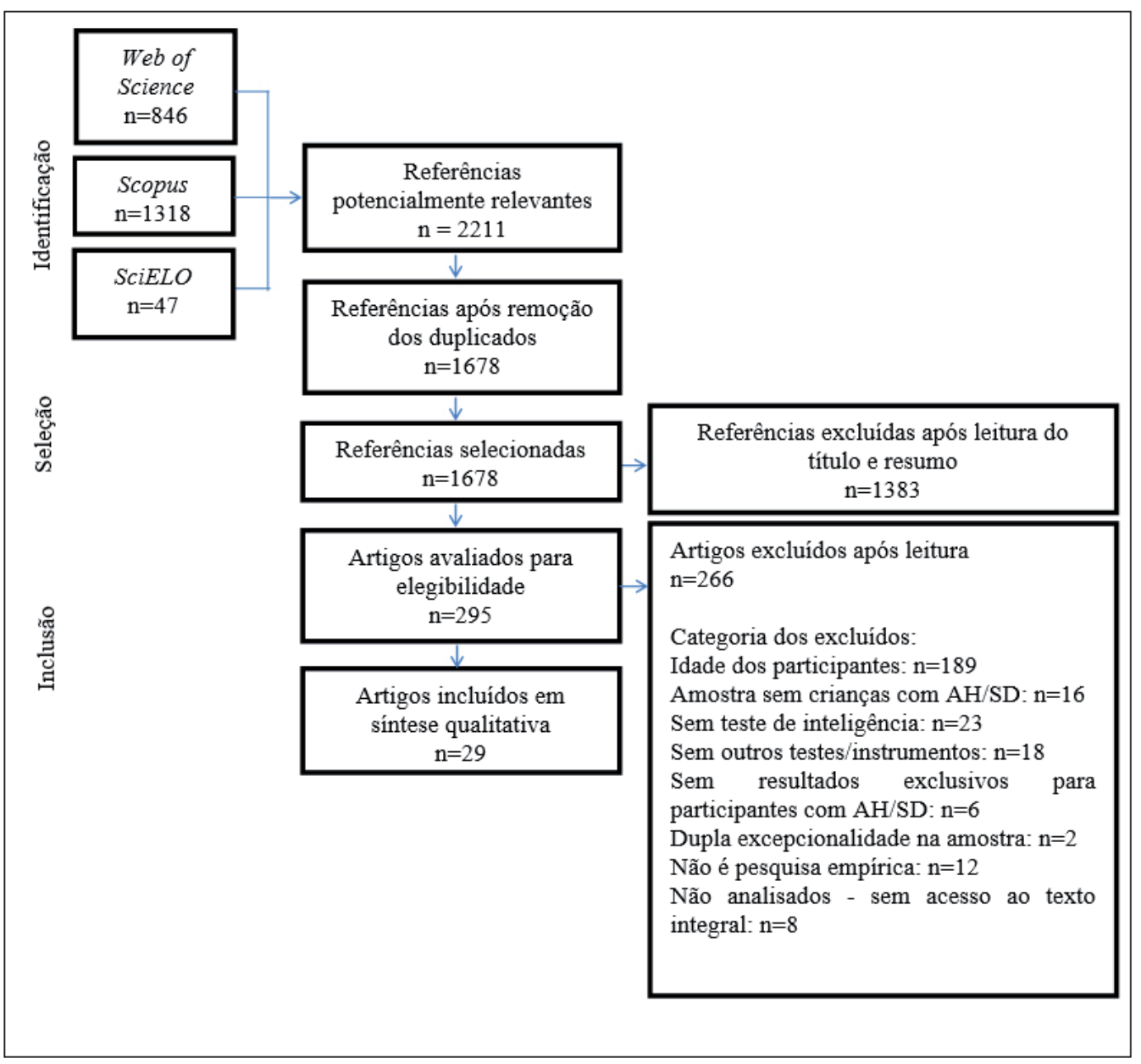

Nota. $\mathrm{n}=$ número de artigos . 


\subsection{Coleta E ANÁlise de dAdos}

Feita a seleção dos artigos relevantes, foi então realizada a extração dos dados relativos à referência do artigo, país da coleta de dados e da universidade onde foi desenvolvido, objetivo, participantes, idade dos participantes, se recebiam atendimento especializado, teste de inteligência utilizado na avaliação, outros instrumentos utilizados na avaliação, QI dos participantes, área de habilidade/talento e principais achados. Em seguida, os artigos foram agrupados em categorias de acordo com o objetivo de cada um.

\section{Resultados E discussão}

A síntese dos resultados é a narrativa. Os dados relacionados à referência dos artigos selecionados e ao país de realização da pesquisa são apresentados na Tabela 1. Vale destacar que cinco artigos não expuseram onde aconteceu a coleta dos dados, apenas há a informação referente à universidade que realizou a pesquisa. Assim, cinco pesquisas foram realizadas em instituiçóes da Holanda, cinco da China, quatro da Austrália, três da Espanha, três da Turquia, três dos Estados Unidos da América, duas do Canadá, duas da Alemanha, uma do México e uma de Singapura. Considerando os critérios de elegibilidade definidos, nenhum artigo brasileiro foi incluído.

\section{Tabela 1}

Lista dos artigos selecionados

\begin{tabular}{|c|c|c|c|}
\hline \multirow{2}{*}{\multicolumn{2}{|c|}{ Artigo $\quad$ Referência }} & \multicolumn{2}{|c|}{ País } \\
\hline & & Coleta & Univercidade \\
\hline 1 & Berg e McDonald (2018) & - & Canadá \\
\hline 2 & Calero et al. (2011) & Espanha & Espanha \\
\hline 3 & Dağlioğlu e Suveren (2013) & Turquia & Turquia \\
\hline 4 & Ersoy et al. (2019) & Turquia & Turquia \\
\hline 5 & Garces-Bacsal (2010) & Filipinas & Singapura \\
\hline 6 & Grant (2013) & - & Austrália \\
\hline 7 & Irby e Floyd (2017) & EUA & EUA \\
\hline 8 & Kroesbergen et al. (2016) & Holanda & Holanda \\
\hline 9 & Li e Shi (2019) & China & China \\
\hline 10 & Liu et al. (2011) & - & China \\
\hline 11 & Mills (2015) & EUA & EUA \\
\hline 12 & Montero-Linares et al. (2013) & - & Espanha \\
\hline 13 & Pilarinos e Solomon (2017) & Canadá & Canadá \\
\hline 14 & Shi et al. (2013) & China & China \\
\hline 15 & Vogelaar et al. (2019) & Holanda & Holanda \\
\hline 16 & Vogelaar e Resing (2017) & Holanda & Holanda \\
\hline 17 & Vogelaar et al. (2017) & Holanda & Holanda \\
\hline 18 & Vogelaar et al. (2016) & Holanda & Holanda \\
\hline 19 & Vogl e Preckel (2014) & Alemanha & Alemanha \\
\hline
\end{tabular}




\begin{tabular}{l|l|l|l}
20 & Walsh et al. (2017) & Austrália & Austrália \\
21 & Walsh e Kemp (2019) & Austrália & Austrália \\
22 & Walsh e Kemp (2012) & - & Austrália \\
23 & Winsler et al. (2013) & EUA & EUA \\
24 & Zhang, Zhang et al. (2016) & China & China \\
25 & Zhang, He et al. (2016) & China & China \\
26 & Martín-Lobo et al. (2018) & Espanha & Espanha \\
27 & Soto e Tomasini (2018) & México & México \\
28 & Beißert e Hasselhorn (2016) & Alemanha & Alemanha \\
29 & Saranli (2017) & Turquia & Turquia \\
\hline
\end{tabular}

Nota. - = não indicado no artigo; EUA = Estados Unidos da América.

Informaçóes referentes aos grupos de participantes (com AH/SD, sem AH/SD, pais e professores), idade das crianças e se recebiam atendimento especializado são apresentadas na Tabela 2. Dentre os artigos, 48,2\% têm grupos de participantes com e sem AH/SD.

\section{Tabela 2}

Informaçöes gerais sobre os participantes

\begin{tabular}{|c|c|c|c|c|c|c|}
\hline \multirow{2}{*}{$\frac{8}{2}$} & \multicolumn{4}{|c|}{ Grupos de participantes } & \multirow{2}{*}{ Idade } & \multirow{2}{*}{ Atendimento Especializado* } \\
\hline & $\begin{array}{l}\text { Com } \\
\text { AH/SD }\end{array}$ & $\begin{array}{c}\text { Sem } \\
\text { AH/SD }\end{array}$ & Pais & Prof. & & \\
\hline 1 & 23 & 28 & - & - & 5 a 8 & Não indica \\
\hline 2 & 64 & 63 & - & - & 7 a 11 & $\begin{array}{l}\text { Aceleração escolar; educação complementar na } \\
\text { sala de aula regular }\end{array}$ \\
\hline 3 & 50 & - & ND & ND & 5 a 6 & Não indica \\
\hline 4 & 10 & - & 10 & - & 11 & Science and Art Center \\
\hline 5 & 22 & - & - & - & 4 a 9 & Escolas para alunos superdotados \\
\hline 6 & 7 & - & $\mathrm{ND}$ & $\mathrm{ND}$ & 4 a 5 & Não indica \\
\hline 7 & 36 & - & - & - & 8 a 11 & Educação Especial para superdotação intelectual \\
\hline 8 & 35 & 34 & - & - & 6 a 8 & Não indica \\
\hline 9 & 80 & 104 & - & - & 8 a 11 & $\begin{array}{l}\text { Gifted Experiment Class: enriquecimento curri- } \\
\text { cular }\end{array}$ \\
\hline 10 & 15 & 13 & - & - & 8 a 10 & Sala de aula para superdotados: Gifted Youth Class \\
\hline 11 & 43 & - & - & - & 7 a 11 & Sala de aula de educação para alunos com AH/SD \\
\hline 12 & 25 & 25 & - & - & 6 a 10 & Não indica \\
\hline 13 & 48 & - & 81 & 36 & 7 a 11 & Não indica \\
\hline
\end{tabular}




\begin{tabular}{|c|c|c|c|c|c|c|}
\hline 14 & 48 & - & - & - & 10 & Grupo de enriquecimento curricular \\
\hline 15 & 50 & 98 & - & - & 9 a 10 & Programas educacionais para AH/SD \\
\hline 16 & 40 & 95 & - & - & 9 a 10 & Educação especial para AH/SD \\
\hline 17 & 45 & 68 & - & - & 7 a 8 & Educação especial para AH/SD \\
\hline 18 & 45 & 68 & - & - & 7 a 8 & Educação especial para AH/SD \\
\hline 19 & 198 & - & - & - & 8 a 11 & $\begin{array}{l}\text { Grupos por habilidade; aceleração e enriqueci- } \\
\text { mento curricular }\end{array}$ \\
\hline 20 & 8 & - & - & - & 3 a 4 & Não indica \\
\hline 21 & 5 & - & - & - & 3 a 4 & Não indica \\
\hline 22 & 1 & - & - & - & 4 & Não indica \\
\hline 23 & 453 & - & - & - & 4 a 9 & Programas educacionais para alunos com AH/SD \\
\hline 24 & 43 & 43 & - & - & 9 a 10 & Turmas para superdotados \\
\hline 25 & 57 & 30 & - & - & 9 a 10 & Turmas para superdotados \\
\hline 26 & 52 & 104 & - & - & 5 a 8 & Não indica \\
\hline 27 & 28 & - & - & - & 8 a 11 & Não indica \\
\hline 28 & 129 & - & - & - & 6 a 8 & Programa de enriquecimento curricular \\
\hline 29 & 1 & 1 & 1 & 1 & 5 & Náo indica \\
\hline
\end{tabular}

Nota . ND = número de participantes não disponível no artigo; - = não contempla; Prof. = Professores; * = atendimentos que os participantes frequentam, mas não necessariamente todos os participantes.

$\mathrm{Na}$ Tabela 3, são apresentados dados relativos aos testes utilizados na avaliação da inteligência. Sobre os resultados do quociente de inteligência dos participantes, os valores variaram de 82 a 160.

\section{Tabela 3}

Testes de inteligência utilizados na avaliação das AH/SD

\begin{tabular}{l|l}
\hline \multicolumn{1}{c|}{ Artigos } & \multicolumn{1}{c}{ Testes de inteligência } \\
\hline $1,2,6,8,12,14,15,16,17,18,20,21$, & Raven \\
$22,24,25,27$ & Brief Intelligence Test \\
2 & $\begin{array}{l}\text { Primary Mental Abilities Test 5-7, Goodenough-Harris Draw-a- } \\
\text {-Person Test }\end{array}$ \\
$4,7,9,10,19,25$ & Teste não foi nomeado \\
$2,5,12,13,23,26$ & WISC-IV ou WISC-R
\end{tabular}




\begin{tabular}{l|l}
$6,9,10,23,24,29$ & $\begin{array}{l}\text { Stanford Binet } \\
\text { Reynolds Intellectual Assessment Scales } \\
7\end{array}$ \\
$9,24,26$ & $\begin{array}{l}\text { WPPSI ou WPPSI-R } \\
\text { Cognitive Abilities Test } \\
\text { Differential ability scales_-II; Kaufman assessment battery for } \\
\text { children; Metropolitan achievement tests, reading diagnostic tests; } \\
\text { APRENDA II } \\
23\end{array}$ \\
$\begin{array}{l}\text { Naglieri Nonverbal Ability Test } \\
\text { Subescalas CFT1 (adaptação parcial - Culture Fair Intelligence } \\
\text { Tests-Scale I) }\end{array}$ \\
\hline
\end{tabular}

Visto a diversidade de instrumentos que não são testes de inteligência que foram utilizados, eles foram agrupados em categorias que são apresentadas na Tabela 4. Apenas nove artigos mencionaram a área de habilidade dos participantes. Em oito deles as habilidades eram intelectuais, e em um eram matemáticas.

\section{Tabela 4}

Outros instrumentos utilizados na identificação e avaliação das AH/SD

\begin{tabular}{l|l}
\hline \multicolumn{1}{c|}{ Categoria } & \multicolumn{1}{c}{ Artigos } \\
\hline Nomeação por professores & $1,3,4,5,8,15,16,17,18,23,27,28,29$ \\
Nomeação por outros profissionais escolares & $12,20,21,22$ \\
Nomeação por pediatra & 5 \\
Nomeação por pais & $2,3,13,15,16,17,18,23$ \\
Pais: entrevistas, escalas & $2,19,22,23,29$ \\
Equipe escolar: avaliações dos profissionais, escalas, entrevistas & $2,7,23,29$ \\
Testes de raciocínio e/ou de habilidades cognitivas & $1,6,9,10,14,20,21,22,24,25,29$ \\
Testes de desempenho e de aptidão escolar & $2,7,11,14,23$ \\
Rendimento escolar & $7,12,14,19$, \\
Criatividade & $8,9,10,14,23,24,26,27$ \\
Talentos e características pessoais & $9,10,14,24,25,27,29$ \\
Condições físicas & $9,10,14,24,25$ \\
Observação de características comportamentais & $9,10,14,19,24,25$ \\
\hline
\end{tabular}

Em relação aos principais achados, inicialmente os artigos foram agrupados em categorias, definidas a partir das similaridades entre os objetivos: relaçóes de aprendizagem e funçóes 
cognitivas; funções executivas; instrumentos de avaliação e processos de identificação; interaçôes sociais e desenvolvimento psicológico; aspectos escolares e educativos. As Tabelas 5, 6, 7, 8 e 9 apresentam os principais achados de cada categoria. Salienta-se o risco de viés entre os dados coletados, já que há artigos que foram publicados por autores que se repetem ou que são originários do mesmo grupo de pesquisadores, havendo o risco de os participantes das pesquisas serem os mesmos, trazendo duplicidade de informações. Não foi possível descartar esse risco, mesmo após a leitura integral dos artigos. O risco de viés é suscitado nos artigos de: Vogelaar et al. (2019) e Vogelaar e Resing (2017); Vogelaar et al. (2017) e Vogelaar et al. (2016); Walsh et al. (2017) e Walsh e Kemp (2019); Zhang, Zhang et al. (2016) e Zhan, He et al. (2016).

\section{Tabela 5}

Principais achados da categoria "Relaçôes de aprendizagem e funçôes cognitivas"

\begin{tabular}{|c|c|}
\hline Artigo & Resultados \\
\hline 1 & $\begin{array}{l}\text { A memória de trabalho visuo-espacial e a memória de curto prazo verbal como fatores preditores da } \\
\text { superdotaçáo na matemática. Entre crianças superdotadas e típicas não houve diferenças em relação } \\
\text { ao controle inibitório. }\end{array}$ \\
\hline 2 & $\begin{array}{l}\text { Aprendem mais e melhor nas distintas tarefas, tendo desempenho superior em memória visual, racio- } \\
\text { cínio verbal e construção perceptual. }\end{array}$ \\
\hline 9 & Diferença significativa nos índices de inteligência fluída, maior autoestima em crianças com AH/SD. \\
\hline 11 & $\begin{array}{l}\text { Alunos que estudavam em salas de aula para superdotados tiveram desempenho acima da média em } \\
\text { testes de vocabulário, comparados com alunos com AH/SD de salas de aula comuns. }\end{array}$ \\
\hline 12 & $\begin{array}{l}\text { Diferença significativa no desempenho em tarefa verbal de atenção sustentada, memória de trabalho } \\
\text { e no potencial de automatizaçáo de tarefas. }\end{array}$ \\
\hline 15 & Ser superdotado e receber treinamento está positivamente associado ao desempenho na tarefa final. \\
\hline 20 & Pensamento de nível superior e extenso conhecimento sobre as coisas. \\
\hline 26 & $\begin{array}{l}\text { Diferenças no funcionamento neuropsicológico de alunos com AH/SD nas habilidades visuais, audi- } \\
\text { tivas, motoras, nos níveis de integração sensorial e tátil, na memória de curto e longo prazo. }\end{array}$ \\
\hline
\end{tabular}

\section{Tabela 6}

Principais achados da categoria "Funçôes executivas"

\begin{tabular}{|c|c|}
\hline Artigo & Resultados \\
\hline 4 & $\begin{array}{l}\mathrm{Na} \text { tomada de decisão consideram o assunto em questão, os aspectos positivos e negativos e com } \\
\text { apoio nas experiências anteriores, assumindo a responsabilidade para as consequências da decisão. }\end{array}$ \\
\hline 10 & $\begin{array}{l}\text { Supressão das informaçóes irrelevantes ou repetitivas de forma mais eficaz e uma função neural mais } \\
\text { madura para a entrada sensorial. }\end{array}$ \\
\hline 14 & $\begin{array}{l}\text { Crianças que recebem educação de enriquecimento curricular concentraram melhor a atenção, dis- } \\
\text { tinguiram estímulos-alvo de distratores e também controlaram impulsos melhor em comparação às } \\
\text { que recebem somente educação regular. }\end{array}$ \\
\hline 16 & As crianças superdotadas não superaram seus pares sem superdotação na resolução de analogias. \\
\hline
\end{tabular}


17

24

As crianças superdotadas não mostraram mais progressão do que as crianças típicas em tarefa de raciocínio analógico, após receber treinamento.

Ao enfrentar uma tarefa atencional e um estímulo visual inesperado simultaneamente, tiveram melhor desempenho na tarefa atencional e foram mais suscetíveis a detectar o estímulo inesperado, em comparação às crianças sem superdotação.

Melhor desempenho em tempo de reação e em tarefas de atenção em comparação às crianças típicas.

\section{Tabela 7}

Principais achados da categoria "Instrumentos de avaliação e processos de identificação"

\begin{tabular}{|c|c|}
\hline Artigo & Resultados \\
\hline 3 & $\begin{array}{l}\text { Das crianças nomeadas por professores e/ou pais, } 44,3 \% \text { foram identificadas como superdotadas. } \\
\text { Elas tiveram desempenho superior ao esperado por seus pais e professores nas áreas de liderança e } \\
\text { performance geral. Os pais foram mais propensos, em relaçáo aos professores, a subestimar o desem- } \\
\text { penho da criança. }\end{array}$ \\
\hline 7 & Variaçôes nas características das respostas e no desempenho das crianças nos testes de QI. \\
\hline 18 & $\mathrm{Na}$ resolução das analogias não mostraram mais progressão na precisão do que seus pares típicos. \\
\hline 23 & $\begin{array}{l}\text { Meninos negros que entraram no programa de superdotados tinham habilidades cognitivas altas aos } \\
\text { quatro anos e eram aqueles que receberam as notas mais altas de seus professores no ano do Pré II. }\end{array}$ \\
\hline 27 & $\begin{array}{l}\text { O tempo gasto pelas mães para realizar tarefas com os filhos e atividades extracurriculares estão nega- } \\
\text { tivamente associadas ao autoconceito acadêmico das crianças. }\end{array}$ \\
\hline 29 & $\begin{array}{l}\text { Desenvolvimento superior à idade nos testes de linguagem, desenvolvimento e inteligência; no en- } \\
\text { tanto, apresenta problemas sociais e emocionais com os pares. }\end{array}$ \\
\hline
\end{tabular}

Nota. QI = Quociente de Inteligência.

\section{Tabela 8}

Principais achados da categoria "Interaçóes sociais e desenvolvimento psicológico"

\begin{tabular}{c|l} 
Artigo & \multicolumn{1}{|c}{ Resultados } \\
\hline 8 & $\begin{array}{l}\text { As preocupaçôes que emergiram nos relatos das crianças, ao responderem um teste projetivo, en- } \\
\text { volvem temas sobre as relaçôes familiares, percepçóes sobre a escola e inteligência, caracterização de } \\
\text { heróis, relaçóes entre pares e preocupaçóes relacionadas a Deus eà espiritualidade. Crianças de escolas } \\
\text { públicas referem mais aspectos de resiliência, enquanto as de escolas privadas expressam aspectos } \\
\text { relacionados à criatividade e à imaginação. }\end{array}$ \\
$\begin{array}{l}\text { Não diferem de crianças típicas nos sentimentos sobre competência escolar, comportamento, prazer } \\
\text { escolar e nos problemas externalizantes e habilidades pró-sociais. Demonstram baixa autoestima e } \\
\text { baixa aceitação social, mas menos problemas internalizantes. Crianças com alta expressão de cria- } \\
\text { tividade tiveram escores baixos em autoestima, competência escolar, aceitação social, conduta, ha- } \\
\text { bilidades pró-sociais e prazer escolar e escores altos em problemas internalizantes e externalizantes, } \\
\text { comparadas às crianças superdotadas com menor expressão de criatividade. }\end{array}$
\end{tabular}


Correlação positiva entre o estilo parental autoritário das mães e pontuaçôes das crianças na escala

13

de status intelectual e escolar. Correlação negativa entre o estilo parental permissivo das mães e os escores das crianças na escala de status intelectual e escolar, de felicidade e satisfação e escore total de autoconceito. Com os pais, há correlação negativa entre o estilo parental permissivo e as pontuaçôes totais do autoconceito.

Alunos de classes de superdotados mostraram interesse estável pela escola e nas classes regulares o interesse diminuiu. A relação aluno-professor deteriorou nas classes regulares e permaneceu constante nas classes para superdotados.

\section{Tabela 9}

Principais achados da categoria "Aspectos escolares e educativos"

\begin{tabular}{|c|c|}
\hline Artigo & Resultados \\
\hline 6 & $\begin{array}{l}\text { A continuidade no processo de transição estava presente quando o novo ambiente escolar continha } \\
\text { elementos familiares ao modo de aprendizagem na família. As relaçóes com o educador dáo maior } \\
\text { suporte ao aluno quando combinam segurança emocional e altos níveis de estimulação intelectual. }\end{array}$ \\
\hline 21 & Perguntas de ordem superior resultaram na produção de linguagem mais sintaticamente sofisticada. \\
\hline 22 & $\begin{array}{l}\text { As respostas às perguntas de nível inferior eram simples e diretas, somente nas perguntas mais com- } \\
\text { plexas a criança foi capaz de demonstrar plenamente suas habilidades verbais e cognitivas. }\end{array}$ \\
\hline
\end{tabular}

Dentre os artigos, 17 indicaram que os participantes recebem algum tipo de atendimento especializado na educação por terem AH/SD. No Brasil, a Lei de Diretrizes e Bases da Educaçáo Nacional - Lei n 9.394, de 20 de dezembro de 1996 - dá a garantia de currículos, métodos, técnicas e recursos educativos adequados às necessidades dos alunos com AH/SD e, também, garante o acesso desses alunos ao Atendimento Educacional Especializado (AEE) no contraturno escolar. Essas garantias são reforçadas na Política Nacional da Educação Especial na Perspectiva da Educação Inclusiva (PNEEPEI, 2008), que reafirma os alunos com AH/SD dentro da demanda da educação especial.

Sobre a avaliação das AH/SD, em relação à inteligência, foram elencados 14 diferentes testes utilizados. Dentre os testes mais mencionados estáo os testes das matrizes progressivas de Raven, citado em 16 artigos, os testes das escalas Wechsler foram utilizados na avaliaçáo dos participantes de oito dos artigos, e o teste Stanford-Binet foi utilizado em seis dos artigos selecionados. Kaufman e Sternberg (2008) discorrem que a inteligência não é um fator único que determina a existência de altas habilidades/superdotaçáo. Nesse sentido, diversos instrumentos de avaliaçáo, além dos testes de inteligência, foram encontrados nos artigos. Verificou-se que os instrumentos mais utilizados foram os de nomeaçấo por professores $(n=13)$, testes de raciocínio e/ou habilidades cognitivas $(n=11)$, de nomeação por pais $(n=8)$ e de investigação da criatividade $(\mathrm{n}=8)$.

$\mathrm{Na}$ categoria "Relaçóes de aprendizagem e funçóes cognitivas", os principais resultados indicam diferenças no funcionamento neuropsicológico de crianças com AH/SD. Elas apresentaram melhor desempenho, em comparação às crianças típicas, nas habilidades visuais, 
auditivas e motoras, nos níveis de integração sensorial e tátil e na memória de curto e longo prazo (Martin-Lobo et al., 2018). O artigo de Montero-Linares et al. (2013) traz dados que demonstram diferença estatisticamente significativa entre crianças com AH/SD e típicas no desempenho em tarefa verbal de atenção sustentada e memória de trabalho, bem como no potencial de automatização de tarefas. Verificou-se, também, em comparação às crianças sem AH/SD, desempenho superior nos domínios de memória visual, raciocínio verbal, construção perceptual (Calero et al., 2011) e nos índices de inteligência fluída (Li \& Shi, 2019), que vai ao encontro do achado de Berg e McDonald (2018). Na pesquisa de Li e Shi (2019), as crianças com superdotação tiveram maior autoestima e as crianças típicas tiveram melhor desempenho em tarefa de baixa impulsividade. $\mathrm{O}$ autocontrole da ação impulsiva é um dos aspectos que envolvem a função de controle inibitório (Diamond, 2013) e no artigo de Berg e McDonald (2018) não foram encontradas diferenças para controle inibitório entre as crianças com e sem AH/SD. A respeito do desempenho acadêmico, o artigo de Vogelaar et al. (2019) apresenta que o estímulo e a instrução dada às crianças são importantes para o desempenho nas tarefas e ser superdotado e ter sido treinado está positivamente associado ao desempenho na tarefa final. As pesquisas de Mills (2015) e Walsh et al. (2017) exploraram aspectos de linguagem e obtiveram resultados com desempenho elevado das crianças com superdotação. Esse achado é corroborado pelo estudo de revisão da literatura feito por Attoni et al. (2020), que analisou características da linguagem de crianças com AH/SD e verificou elevada habilidade de compreensão oral e escrita, rico vocabulário e interesse precoce pela leitura.

Ampliando os aspectos relativos às funçóes cognitivas, destacam-se também as funçóes executivas. Dos achados dessa categoria, Vogelaar e Resing (2017) e Vogelaar et al. (2017) evidenciam que as crianças superdotadas náo superaram seus pares sem superdotaçáo na resolução das analogias. Ersoy et al. (2019) verificaram que elas apresentam habilidade para tomar decisôes. Segundo afirmado por Diamond (2013), a memória operacional tem importante papel no processo de tomada de decisão, em consonância, portanto, com os resultados obtidos por Berg e McDonald (2018), Martín-Lobo et al. (2018) e Montero-Linares et al. (2013), os quais demonstraram elevado desempenho dos participantes em tarefas de memória operacional. Esse desempenho também foi investigado por Aubry et al. (2021), que identificaram nas crianças superdotadas maior capacidade de memória operacional e que elas conseguem resolver problemas rapidamente, em comparação às crianças típicas. Liu et al. (2011) relatam que as crianças superdotadas suprimiram informaçóes irrelevantes ou repetitivas de forma mais eficaz e, também, apresentaram ter uma função neural mais madura para a entrada sensorial. Zhang, Zhang et al. (2016) e Zhang, He et al. (2016) constataram maior sensibilidade para perceber estímulos inesperados e melhor desempenho em tarefa atencional. O ambiente educacional pode ser um fator interveniente no desempenho das crianças, visto que Shi et al. (2013) identificaram que a as crianças que recebiam enriquecimento curricular tiveram melhor desempenho na ação, inibição e sensibilidade perceptual, conseguiram concentrar melhor a atenção e, por mais tempo, distinguir estímulos-alvo de distratores e, também, controlar melhor seus impulsos. Destacam-se os benefícios da oferta de educação diferenciada para as AH/SD.

Conhecer aspectos da cognição é importante, pois eles contribuem, junto a outros aspectos, no processo de identificação das crianças com altas habilidades/superdotação (Piske et al., 2017). Na categoria "Instrumentos de avaliação e processos de identificação", os achados de 
Winsler et al. (2013) indicam singularidades na identificação da superdotação em meninos negros, ressaltando fatores culturais e ambientais. Sobre características dos testes e o desempenho das crianças, Vogelaar et al. (2016) utilizaram testagem dinâmica e verificaram que as crianças superdotadas não mostraram mais progressão que seus pares típicos. Irby e Floyd (2017) constataram variaçóes no desempenho das crianças, que obtiveram diferentes resultados em diferentes testes de inteligência. Na identificação de AH/SD, a nomeação por pais e professores é bastante utilizada (Virgolim, 2007); nesse sentido, os artigos de Daglioglu e Suveren (2013) e Soto e Tomasini (2018) refletem perspectivas familiares na expressão da superdotação. Também abordando aspectos familiares nas AH/SD, o estudo de Piske et al. (2015) demonstrou preocupação dos pais em relação ao processo de ensino-aprendizagem dos filhos, principalmente devido às dificuldades emocionais, sociais e cognitivas, bem como dificuldade na compreensão das características das altas habilidades que seus filhos apresentam.

O desenvolvimento assíncrono de uma criança de cinco anos e três meses com superdotação foi investigado por Saranli (2017). Nesse sujeito, as etapas de desenvolvimento relacionadas à linguagem e ao desenvolvimento motor ocorreram precocemente e ele obteve desenvolvimento superior à idade nos testes de cognição, mas apresentou dificuldades nas interações sociais. Esses achados estão em conformidade com Terrassier (2009), que discorre sobre uma dissincronia no desenvolvimento global dessas crianças.

$\mathrm{Na}$ categoria "Interaçóes sociais e desenvolvimento psicológico", o artigo de Beißert e Hasselhorn (2016) elucida que as competências de inteligência não puderam explicar as diferenças no desenvolvimento moral. Garces-Bacsal (2010) apresenta que os relatos e as preocupaçóes de crianças da escola pública trazem à tona aspectos de resiliência, enquanto as crianças da escola privada expressam relatos que envolvem criação e imaginação. Nesse sentido, Stoltz et al. (2015) referem que o ambiente é fundamental para o desenvolvimento do processo criativo, pois é nele que a criança vive experiências que estimulam a curiosidade, o desejo de aprender, a fantasia e a imaginação. Os achados de Vogl e Preckel (2014) expressam que alunos de classes de superdotados mostraram interesse estável pela escola, enquanto nas classes regulares o interesse pela escola diminuiu. De acordo com Kroesbergen et al. (2016), crianças com AH/SD demonstraram baixa autoestima e baixa aceitação social em relação às crianças típicas. Esse achado é contrário ao de Li e Shi (2019), que verificaram maior autoestima nas crianças com AH/SD. Os achados de Pilarinos e Solomon (2017) apresentam uma correlação positiva estatisticamente significativa entre o estilo parental autoritário das mães e pontuações mais altas das crianças na escala de status intelectual e escolar. Destacam-se aqui indagaçóes para outros estudos na área, visto que adotar um estilo parental autoritativo é o recomendado para a educação saudável das crianças (Weber et al., 2004).

Sobre os "Aspectos escolares e educativos", Grant (2013) verificou que, no processo de transição escolar, a influência positiva foi encontrada na semelhança dos ambientes de aprendizagem em casa e na escola e que as relaçôes com o educador devem combinar segurança emocional e altos níveis de estimulação intelectual. Evidenciam-se nos artigos de Grant (2013), Walsh e Kemp $(2012,2019)$ aspectos da interação do aluno com a escola, que podem contribuir ou não para a aprendizagem e desempenho. A interação entre professor e aluno é presente nos artigos de Walsh e Kemp $(2012,2019)$ e os resultados demonstraram que diante de perguntas complexas do professor as crianças responderam com uma linguagem mais sofisticada. 
Nesse sentido, é fundamental destacar a importância do ambiente educativo diferenciado para os alunos com AH/SD, de modo que atenda às demandas específicas deles no contexto escolar (Cupertino, 2008; Renzulli, 2005; Winner, 1998). Ao abordar os principais achados, é necessário destacar que esta pesquisa apresenta limitaçóes. No que se refere aos artigos e aos desfechos podem ser elencados aspectos como artigos de autores que se repetem ou que são originários dos mesmos grupos de pesquisa e instituições de realização, que podem trazer duplicidade de resultados, uma vez que não há a certeza se os participantes são de amostras diferentes ou não. Outra limitação é que os resultados, mesmo que agrupados em categorias por semelhança, não podem ser generalizados, pois são advindos de pesquisas que utilizaram métodos diferentes. As limitações, no nível da revisão, são principalmente a obtenção incompleta dos artigos existentes, visto que não foi possível acessar oito dos artigos potencialmente relevantes, e, também, é preciso considerar que o uso de outras bases de dados e outras palavras-chave poderiam, talvez, indicar outros artigos. Sendo os principais achados relatados a partir de uma síntese narrativa, há também de se considerar a possibilidade de produzir outras sínteses para os resultados.

\section{Conclusóes}

Os resultados apresentam uma variedade de características sobre as crianças superdotadas, confirmando o caráter heterogêneo das AH/SD. No entanto, a maioria das pesquisas refere-se a aspectos da cognição e da identificação e avaliação, deixando aparente uma lacuna nos estudos sobre o desenvolvimento psicológico, autonomia, relaçóes interpessoais e familiares, dentre outros. Evidenciam-se as diferenças, em comparação às crianças típicas, no funcionamento neuropsicológico de crianças com AH/SD, nas áreas de integração sensorial, habilidades visuais, auditivas, memória de trabalho, de curto e longo prazo, raciocínio matemático, verbal e em leitura, inteligência fluida, atenção, aprendizagem e organização perceptual (Berg \& McDonald, 2018; Calero et al., 2011; Li \& Shi, 2019; Liu et al., 2011; Martin-Lobo et al., 2018; Montero-Linares et al., 2013; Zhang, He et al., 2016; Zhang, Zhang et al., 2016). Sobre aspectos da relação familiar, de um modo geral, nas suas áreas de habilidade, as crianças superdotadas apresentam desempenho mais elevado que as expectativas de seus pais (Daglioglu \& Suveren, 2013), bem como demonstram estratégias adequadas de tomada de decisão e recebem suporte de seus pais para isso (Ersoy et al., 2019). As interaçóes na família nuclear têm relaçóes com o comportamento adaptativo, com o rendimento escolar e autoconceito das crianças (Pilarinos \& Solomon, 2017).

Receber educação diferenciada é um fator que reflete benefícios sociais e acadêmicos (Grant, 2013; Vogl \& Preckel, 2014; Walsh \& Kemp, 2019). É essencial que os professores reconheçam as habilidades de seus alunos e ofereçam atividades estimulantes ao nível cognitivo de cada um, assim como é fundamental a garantia do acesso à educação inclusiva. Dessa forma, conhecer as características de crianças com AH/SD contribui, no âmbito escolar, para a identificação desses alunos e a oferta da educação preconizada na PNEEPEI (2008). Visto que o conhecimento que os professores têm acerca dos alunos com superdotação ainda é insuficiente para a promoção de práticas educativas adequadas (Bahiense \& Rossetti, 2014; Barreto \& Mettrau, 2011), este estudo apresenta um importante conhecimento para a área da Educação Especial. Esta pesquisa contribui para a visibilidade sobre as características de crianças com $\mathrm{AH} / \mathrm{SD}$, com um enfoque geral e amplo. Sugere-se que futuras pesquisas busquem explorar as 
características das crianças nas diferentes áreas de habilidades. Também se ressalta a importância de estudos que abarquem outras faixas etárias, já que cada etapa de desenvolvimento tem suas próprias peculiaridades. Considerando a variedade de resultados e os métodos de pesquisa nessa área, a realização de mais pesquisas promovendo a replicação dos resultados, com o mesmo objetivo e método, fortalecerá os resultados de pesquisas que visam caracterizar pessoas com superdotação.

\section{REFERÊNCIAS}

Attoni, T., Coelho, R., Martins, R., Lemos, L., Fernandes, L., Francis, T., Fernandes, S., \& Fidelis, K. (2020). Os aspectos da linguagem de crianças com altas habilidades/superdotação: revisão integrativa da literatura. Revista CEFAC. 22(6), 1-6. https://doi.org/10.1590/1982-0216/20202269320

Aubry, A., Gonthier, C., \& Bourdin, B. (2021). Explaining the high working memory capacity of gifted children: contributions of processing skills and executive control. Acta psychologica, 218, 1-12. https://doi.org/10.1016/j.actpsy.2021.103358

Bahiense, T. R. S., \& Rossetti, C. B. (2014). Altas habilidades/superdotação no contexto escolar: percepçôes de professores e prática docente. Revista Brasileira de Educação Especial, 20(2), 195-208. https://doi.org/10.1590/S1413-65382014000200004

Barreto, C. M. P. F., \& Mettrau, M. B. (2011). Altas habilidades: uma questão escolar. Revista Brasileira de Educação Especial, 17(3), 413-426. https://doi.org/10.1590/S1413-65382011000300005

Beißert, H. M., \& Hasselhorn, M. (2016). Individual differences in moral development: Does intelligence really affect children's moral reasoning and moral emotions? Frontiers in Psychology, 7, 1-10. https://doi.org/10.3389/fpsyg.2016.01961

Berg, D. H., \& McDonald, P. A. (2018). Differences in mathematical reasoning between typically achieving and gifted children. Journal of Cognitive Psychology, 30(3), 281-291. https://doi.org/10. 1080/20445911.2018.1457034

Calero, M. D., Belen, G-M., M., \& Robles, M. A. (2011). Learning potential in high IQ children: The contribution of dynamic assessment to the identification of gifted children. Learning and Individual Differences, 21, 176-181. https://doi.org/10.1016/j.lindif.2010.11.025

Civil rights data collection. (2016). Department of Education. United States of America. https://ocrdata. ed.gov/StateNationalEstimations/Estimations_2015_16

Costa, A. B., \& Zoltowski, A. P. C. (2014). Como escrever um artigo de revisão sistemática. In S. H. Koller, M. C. P. de P. Couto, \& J. V. Hohendorff (Orgs.), Manual de produção cientifica (1a ed., pp. 55-70). Penso.

Cupertino, C. M. B. (2008). Um olhar para as altas habilidades: construindo caminhos. Secretaria da Educação do Estado de São Paulo. http://cape.edunet.sp.gov.br/cape_arquivos/Um_Olhar_Para_ As_Altas_habilidades_2\%C2\%B0_Edi\%C3\%A7\%C3\%A3o.pdf

Dağlioğlu, H. E., \& Suveren, S. (2013). The role of teacher and family opinions in identifying gifted kindergarten children and the consistence of these views with children's actual performance. Educational Sciences: Theory \& Practice, 13(1), 444-453. www.edam.com.tr/estp

Diamond, A. (2013). Executive Functions. Annual reviews psychology, 64, 135-168. https://doi. org/10.1146/annurev-psych-113011-143750 
Ersoy, E., Ogurlu, U., \& Aydin, H. (2019). Gifted students' and their parents' perceptions of decision making processes: a Turkish case. Springer Nature, 50, 403-421 https://doi.org/10.1007/s10780019-09357-1

Garces-Bacsal, R. M. (2010). Tales gifted children tell: exploring PTAT responses as pathways to socio-affective concerns. Gifted Child Quarterly, 54(2), 138-151. https://doi. org/10.1177/0016986209358616

Grant, A. (2013). Younggifted children transitioning into preschool and school: What matters? Australasian Journal of Early Childhood, 38(2), 23-31. https://doi.org/10.1177/183693911303800204

Instituto Nacional de Estudos e Pesquisas Educacionais Anísio Teixeira. (2020). Sinopse Estatística da Educação Básica 2020. INEP. https://www.gov.br/inep/pt-br/areas-de-atuacao/pesquisasestatisticas-e-indicadores/censo-escolar/resultados

Irby, S. M., \& Floyd, R. G. (2017). The exchangeability of brief intelligence tests for children with intellectual giftedness: illuminating error variance components' influence on IQS. Psychol Schs, 54, 1064-1078. https://doi.org/10.1002/pits.22068

Kaufman, S. B., \& Sternberg, R. J. (2008). Conceptions of giftedness. In S. I. Pfeiffer (Org.), Handbook of giftedness in children: psychoeducational theory, research, and best practices (1a ed., pp. 71-91). Springer. https://doi.org/10.1007/978-3-319-77004-8

Kroesbergen, E. H., van Hooijdonk, M., Viersen, S. V., Middel-Lalleman, M. M. N., \& Reijnders, J. J. W. (2016). The psychological well-being of early identified gifted children. Gifted Child Quarterly, 6O(1), 16-30. https://doi.org/10.1177/0016986215609113

Lei n ${ }^{\circ}$ 9.394, de 20 de dezembro de 1996. Diretrizes e Bases da Educação Nacional. http://www.planalto. gov.br/ccivil_03/leis/19394.htm

Lei no 8.069, de 13 de julho de 1990. Estatuto da Criança e do Adolescente. http://www.planalto.gov.br/ ccivil_03/leis/18069.htm

Li, D., \& Shi, J. (2019). Fluid intelligence, trait emotional intelligence and academic performance in children with different intellectual levels. High Ability Studies, 32(1), 51-69. https://doi.org/10.10 80/13598139.2019.1694493

Liu, T., Xiao, T., Shi, J., \& Zhao, L. (2011). Sensory gating, inhibition control and child intelligence: an event-related potentials study. Neuroscience, 189, 250-257. https://doi.org/10.1016/j. neuroscience.2011.05.009

Martín-Lobo, P., Santiago-Ramajo, S., \& Vergara-Moragues, E. (2018). Neuropsychological differences among students with learning difficulties, without learning difficulties, and with high capacity. Mind, Brain, and Education, 12(3), 140-154. https://doi.org/10.1111/mbe.12184

Mills, M. T. (2015). Narrative performance of gifted African American school-aged children from low-income backgrounds. American Journal of Speech-Language Pathology, 24, 36-46. https://doi. org/10.1044/2014_AJSLP-13-0150

Montero-Linares, J., Navarro-Guzmán, J. I., \& Aguilar-Villagrán, M. (2013). Procesos de automatización cognitiva en alumnado con altas capacidades intelectuales. Anales de psicologia, 29(2), 454-461. https://doi.org/10.6018/analesps.29.2.123291

Page, M. J., McKenzie, J., E., Bossuyt, P. M., Boutron, I., Hoffmann, T. C., Mulrow, C. D., Shamseer, L., Tetzlaff, J. M., Akl, E. A., Brennan, S. E., Chou, R., Glanville, J., Grimshaw, J. M., Hróbjartsson, A., Lalu, M. M., Li, T., Loder, E. W., Mayo-Wilson, E., McDonald, S., McGuinness, L. A... Moher, 
D. (2021). The PRISMA 2020 statement: an updated guideline for reporting systematic reviews. Research methods and reporting, 372(71), 1-9. https://doi.org/10.1136/bmj.n71

Pfeiffer, S. I. (2015). Gifted students with a coexisting disability: the twice exceptional. Estudos de psicologia, 32(4), 717-727. https://doi.org/10.1590/0103-166X2015000400015

Pilarinos, V., \& Solomon, C. R. (2017). Parenting styles and adjustment in gifted children. Gifted Child Quarterly, 61(1), 87-98. https://doi.org/10.1177/0016986216675351

Piske, F. H. R., Stoltz, T., \& Bahia, S. (2015). Percepções de famílias de superdotados sobre o processo de ensino-aprendizagem: um olhar a partir de Piaget. Schème, 7(2), 78-97. https://doi. org/10.36311/1984-1655.2015.v7n2.p78-97

Piske, F. H. R., Stoltz, T., Camargo, D. de., Vestana, C. L. B., Machado, J. M., Freitas, S. P. de, Dias, C. L., \& Taucei, J. dos R. (2017). Creation process during learning of gifted students: contributions from Jean Piaget. Creative education, 8, 505-513. https://doi.org/10.4236/ce.2017.84039

Piske, F. H. R., Stoltz, T., Vestena, C. L. B., Freitas, S. P. de., Valentim, B. de F. B., Oliveira, C. S. de., Barby, A. A. de O.M., \& Machado, C. L. (2016). Barriers to creativity, identification and inclusion of gifted student. Creative education, 7, 1899-1905. http://dx.doi.org/10.4236/ce.2016.714192

Política nacional da educaçâo especial na perspectiva da educação inclusiva. (2008). Ministério da Educação. http://portal.mec.gov.br/arquivos/pdf/politicaeducespecial.pdf

Renzulli, J. S. (2005). The Three-Ring conception of giftedness: a developmental model for promoting creative productivity. In R. J. Sternberg, \& J. Davidson (Org.), Conceptions of giftedness (2a ed., pp. 217-245). Cambridge University Press. https://doi.org/10.1017/CBO9780511610455

Saranli, A. G. (2017). Eş Zamanlı Olmayan Gelişimin Üstün Yetenekli Çocuklardaki Görünümü Üzerine Bir Örnek Olay Çalışması. Ankara Üniversitesi Eğitim Bilimleri Fakültesi Özel Eğitim Dergisi, 18(1), 89-108. https://doi.org/10.21565/ozelegitimdergisi.300060

Shi, J., Tao, T., Chen, W., Cheng, L., Wang, L., \& Zhang, X. (2013). Sustained attention in intellectually gifted children assessed using a continuous performance test. PLoS ONE, 8(2), 1-8. https://doi. org/10.1371/journal.pone.0057417

Soto, B. I. C., \& Tomasini, G. A. (2018). Children with high capacities: analyses of the relatives' variables involved in the development of the potential. Electronic Journal of Research in Educational Psychology, 16(2), 273-300. https://doi.org/10.25115/ejrep.v16i45.2094

Stoltz, T., Piske, F. H. R., Freitas, M. de F. Q. de., D’Aroz, M. S., \& Machado, J. M. (2015). Creativity in gifted education: contributions from Vygostky and Piaget. Creative education, 6, 64-70. https:// doi.org/10.4236/ce.2015.61005

Terrassier, J. C. (2009). Les enfants intellectuellement precoces. Archives de pédiatrie, 16(12), 16031606. https://doi.org/10.1016/j.arcped.2009.07.019

Virgolim, A. M. R. (2007). Altas habilidades/superdotação: encorajando potenciais. Ministério da Educação, Secretaria de Educação Especial. http://www.dominiopublico.gov.br/download/texto/ me004719.pdf

Vogelaar, B., \& Resing, W. C. M. (2017). Changes over time and transfer of analogy-problem solving of gifted and non-gifted children in a dynamic testing setting. Educational Psychology, 38(7), 898-914. https://doi.org/10.1080/01443410.2017.1409886 
Vogelaar, B., Bakker, M., Elliott, J. G., \& Resing, W. C. M. (2016). Dynamic testing and test anxiety amongst gifted and average-ability children. British Journal of Educational Psychology, 87(1), 75-89. https://doi.org/10.1111/bjep.12136

Vogelaar, B., Bakker, M., Hoogeveen, L., \& Resing, W. C. M. (2017). Dynamic testing of gifted and average-ability children's analogy problem solving: does executive functioning play a role? Psychology in the Schools, 54, 837-851. https://doi.org/10.1002/pits.22032

Vogelaar, B., Resing, W. C. M., Stad, F. E., \& Sweijen, S. W. (2019). Is planning related to dynamic testing outcomes? Investigating the potential for learning of gifted and average-ability children. Acta Psychologic, 196, 87-95. https://doi.org/10.1016/j.actpsy.2019.04.004

Vogl, K., \& Preckel, F. (2014). Full-time ability grouping of gifted students: impacts on social self-concept and school-related attitudes. Gifted Child Quarterly, 58(1), 51-68. https://doi. org/10.1177/0016986213513795

Walsh, R. L., \& Kemp, C. (2012). Evaluating interventions for young gifted children using singlesubject methodology: a preliminary study. Gifted Child Quarterly, 57(2), 110-120. https://doi. org/10.1177/0016986212466259

Walsh, R. L., \& Kemp, C. R. (2019). The effect of higher-order questioning on the complexity of gifted preschoolers' language. The Australasian Journal of Gifted Education, 28(1), 5-22. https://doi. org/10.21505/ajge.2019.0002

Walsh, R., Bowes, J., \& Sweller, N. (2017). Why would you say goodnight to the moon? Response of young intellectually gifted children to lower and higher order questions during storybook. Journal for the Education of the Gifted, 40(3), 220-246. https://doi.org/10.1177/0162353217717032

Weber, L. N. D., Prado, P. M., Viezzer, A. P., \& Brandenburg, O. J. (2004). Identificação de estilos parentais: o ponto de vista dos pais e dos filhos. Psicologia: reflexão e crítica, 17(3), 323-331. https:// doi.org/10.1590/S0102-79722004000300005

Winner, E. (1998). Crianças superdotadas: mitos e realidades. Artmed.

Winsler, A., Karkhanis, D. G., Kim, Y. K., \& Levitt, J. (2013). Being black, male, and gifted in Miami: prevalence and predictors of placement in elementary school gifted education programs. The Urban Review, 45, 416-447. https://doi.org/10.1007/s11256-013-0259-0

Zhang, H., He, Y., Tao, T., \& Shi, J-N. (2016). Intellectually gifted rural-to-urban migrant children's attention. High Ability Studies, 27(2), 193-209. https://doi.org/10.1080/13598139.2016.1242064

Zhang, H., Zhang, X., He, Y., \& Shi, J. (2016). Inattentional blindness in 9 to 10 year-old intellectually gifted children. Gifted Child Quarterly, 60(4), 1-9. http://dx.doi.org/10.1177/0016986216657158-

Recebido em: 13/06/2021

Reformulado em: 03/09/2021

Aprovado em: 05/09/2021 